\title{
P-0632- High glucose promotes smooth muscle cell differentiation of bone marrow stem cells via Erk1/2-TGF $\beta 1$-Smad pathway
}

Meng Jiang ${ }^{1,2}$, Zhenguo Liu², Minxiang Lei ${ }^{1}$

1 Department of Endocrinology, Xiangya Hospital of Central South University, Changsha, China. 2 Department of Medicine, University of Missouri School of Medicine, Columbia, MO, USA.

Background:

Vascular complications are the most serious manifestations and the leading cause of death in patients with type 2 or type 1 diabetes. A prominent feature in the pathology of diabetic vascular disease is the increased number of vascular smooth muscle cells (VSMCs) in the intima of arteries. Recent evidence suggests that the mobilization and recruitment of abundant stem/progenitor cells present in the vessel wall are largely responsible for SMC accumulation in the intima during vascular remodeling such as neointimal hyperplasia and arteriosclerosis. Therefore, understanding the regulatory mechanisms that control SMC differentiation from stem/progenitor cells in diabetes is essential for exploring therapeutic targets for vascular complications. It is well-known that Erk1/2 signaling plays an important role in the differentiation of stem cells. In addition, the role of TGF $\beta 1 / \mathrm{Smad}$ signaling in SMC development is well established. However, the role of high glucose in stem cell differentiation into SMCs has not been clarified.

\section{Aims:}

The present study was to investigate the effects of high glucose on the differentiation of bone marrow stem cells and related mechanism.

\section{Methods:}

Rat multipotent adult progenitor cells (MAPCs) were used as the source of bone marrow stem cells and induced to differentiate in vitro with/without high glucose for up to 2 weeks. Further, MAPCs were mixed in Matrigel and injected under the skin of 8-week old nude mice with/without diabetes. After 2 weeks, plugs were harvested and processed for differentiation assays. We also employed Erk1/2 inhibitor, Erk1/2 siRNA, TGFß1-neutralizing antibody, and Smad inhibitor to study the molecular mechanisms underlying the regulation of Erk1/2 signaling and TGF $\beta 1 /$ Smad signaling by high-glucose. The expression of SMC markers including $\alpha$-SMA, SM22 and CNN1 were determined with western blot, immunofluorescent staining or flowcytometry.

\section{GFP-MAPCS GFP-ERK siRNA-MAPCS}

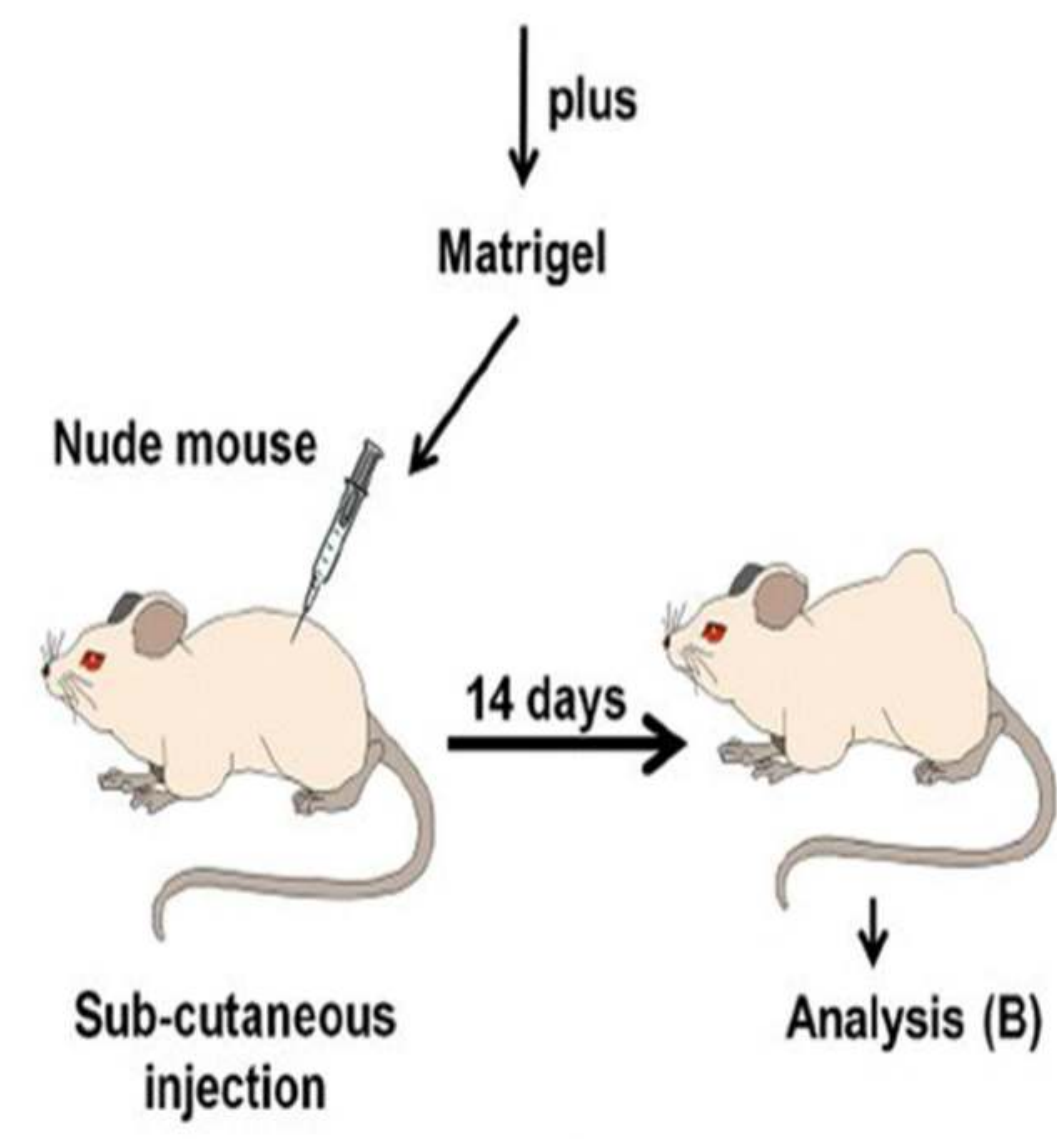

\section{Results:}

The high glucose significantly increased expression of $\alpha$-SMA, SM22, and CNN1 in vitro and in vivo. Treatment with high glucose activated the phosphorylation of Erk1/2 and TGF $\beta 1 /$ Smad pathway in the cells. Treatment with Erk1/2 inhibitor, Erk1/2 siRNA, TGFß1-neutralizing antibody, and Smad inhibitor could reverse the effects of high-glucose on SMC differentiation in vitro and in vivo.

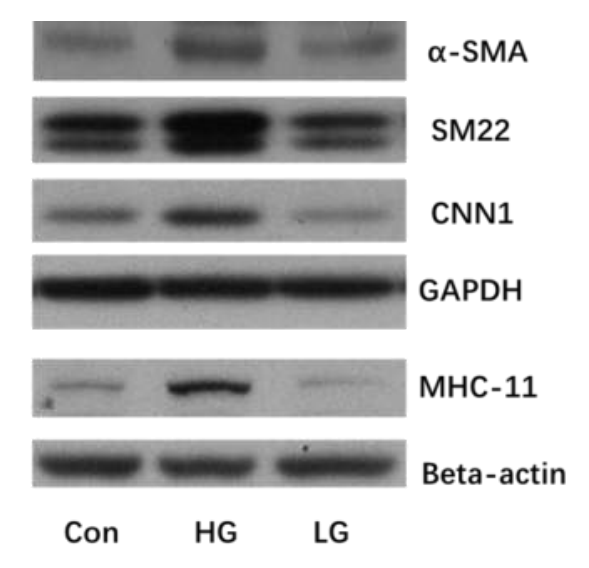

Figure 1 High glucose increased expression of markers of SMCs.

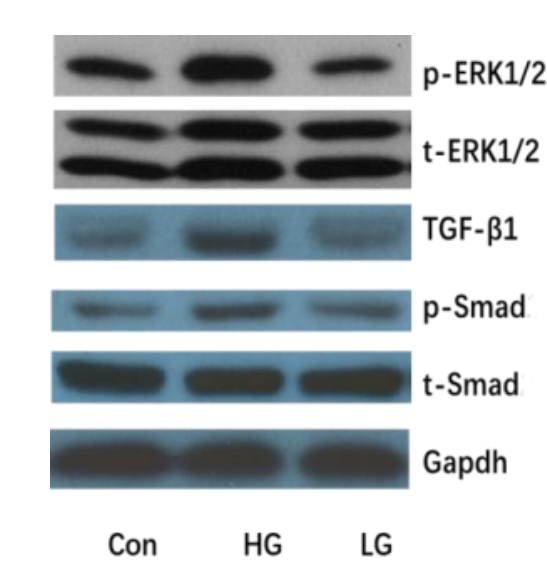

Figure 2 High glucose affected expression of ERK/TGF- $\beta /$ Smad pathway.

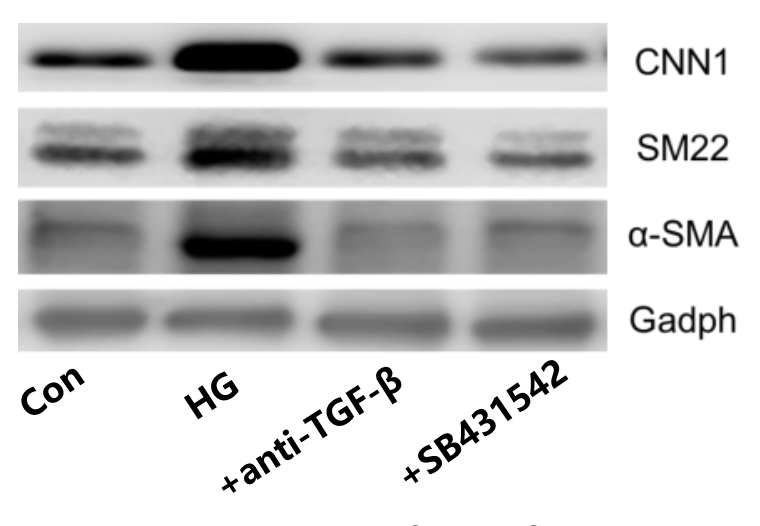

Figure 3 Inhibition of TGF- $\beta$ reversed the effects of high glucose in vitro.

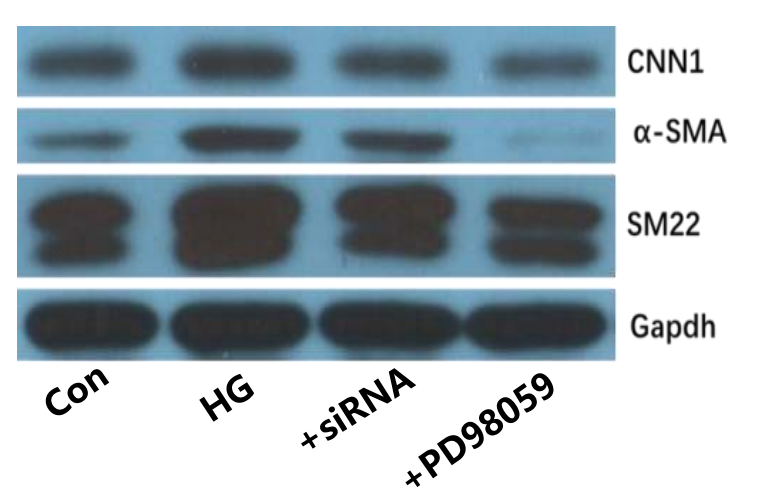

Figure 4 Inhibition of ERK reversed the effects of high glucose in vitro.

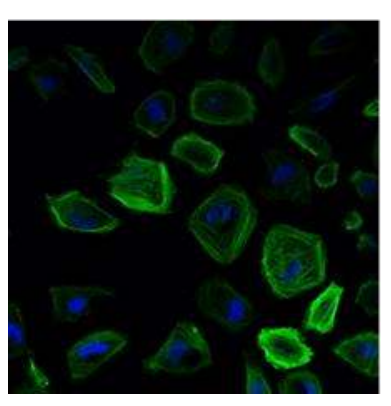

Con

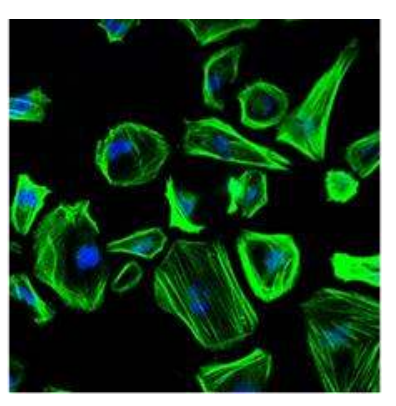

DM

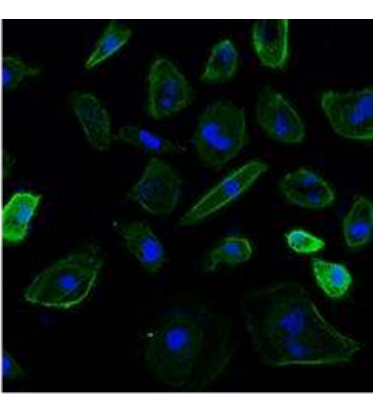

DM+siRNA

Figure 5 Inhibition of ERK reversed the effects of high glucose in vivo.

Discussion:

These data suggest that high glucose could promote the SMC differentiation of bone marrow stem cells by activating Erk1/2-TGF- $\beta$ Smad pathway. Stem cells differentiation appears to be a potential target for the treatment of diabetic vascular complications.

\section{References}

[1] Domingueti CP, Dusse LM, Md C, de Sousa LP, Gomes KB, Fernandes AP. Diabetes mellitus: The linkage between oxidative stress, inflammation, hypercoagulability and vascular complications. J Diabetes Complications. 2016. 30(4): 738-45.

[2] Qi W, Li Q, Liew CW, et al. SHP-1 activation inhibits vascular smooth muscle cell proliferation and intimal hyperplasia in a rodent model of insulin resistance and diabetes. Diabetologia. 2017. 60(3): 585-596.

[3] Shimizu K, Sugiyama S, Aikawa M, et al. Host bone-marrow cells are a source of donor intimal smooth- muscle-like cells in murine aortic transplant arteriopathy. Nat Med. 2001. 7(6): 738-41.

[4] Wang G, Jacquet L, Karamariti E, Xu Q. Origin and differentiation of vascular smooth muscle cells. J Physiol. 2015. 593(14): 3013-30.

[5] Ross JJ, Hong Z, Willenbring B, et al. Cytokine-induced differentiation of multipotent adult progenitor cells into functional smooth muscle cells. J Clin Invest. 2006. 116(12): 3139-49.

[6] Jiang $\mathrm{Y}$, Jahagirdar BN, Reinhardt RL, et al. Pluripotency of mesenchymal stem cells derived from adult marrow. Nature. 2002. 418(6893): 41-9. 